\title{
Bloch oscillations
}

\author{
By Heinrich Kurz ${ }^{1}$, Hartmut G. Roskos ${ }^{1}$, Thomas Dekorsy ${ }^{1}$ \\ AND KLAUS KÖHLER ${ }^{2}$ \\ ${ }^{1}$ Institut für Halbleitertechnik II, Rheinisch-Westfälische Technische Hochschule \\ (RWTH) Aachen, D-52056 Aachen, Germany \\ ${ }^{2}$ Fraunhofer-Institut für Angewandte Festkörperforschung, \\ D-79108 Freiburg, Germany
}

The status quo of the research on Bloch oscillations in semiconductor superlattices is outlined. Bloch oscillations excited by femtosecond laser pulses have been investigated by different optical techniques. They have allowed measurement of the internal electric field changes associated with the charge oscillations, the emitted terahertz electromagnetic radiation and the nonlinear coherent optical response, all directly in the time domain. The main results are summarized and critically reviewed. The potential of Bloch oscillations for practical applications in terahertz radiation sources is assessed.

\section{Introduction}

Bloch oscillations are a manifestation of a specific form of tunnelling in electrically biased superlattice potentials, i.e. coherent tunnelling. With the electronic levels in the periodic potential of the superlattice at, or close to, resonance, the wavefunctions are quasi-delocalized extending across several wells. A superposition of a series of such states forms a wavepacket, the time evolution of which is determined by the phase factors of the states. The wavepacket undergoes a periodic motion, the Bloch oscillation, with the centre of mass tunnelling back and forth through the barriers of the potential.

Bloch oscillations are a quantum-interference phenomenon that can be excited by femtosecond optical pulses. The periodic changes of the dipole moments associated with the charge oscillations are traced by two different time-resolved optical techniques. This paper summarizes the most recent results and identifies areas requiring further research.

\section{Fundamental aspects of Bloch oscillations}

The concept of Bloch oscillations is of prime significance for transport in all crystal structures. Their periodic potentials force carriers principally to perform spatial oscillations (the Bloch oscillations) under the influence of an electric bias field. The problem of the dynamics of electronic wavepackets in electrically biased periodic potentials was addressed as early as 1928 by Bloch soon after the inception of quantum mechanics (Bloch 1928). According to the well-known acceleration theorem, a carrier wavepacket composed of single-band states with a narrow spread of $\boldsymbol{k}$-vectors will 
propagate with a constant velocity through $\boldsymbol{k}$-space when subjected to a uniform electric field $\boldsymbol{F}$. Consequently, the wavepacket, following the energy-band contour in $\boldsymbol{k}$-space, will experience a sign reversal of its real-space velocity when it reaches the first Brillouin-zone boundary (Zener 1934). The wavepacket reverses its propagation direction in real space and begins to travel against the field direction. Thus, carriers experience a dynamical localization. They perform Bloch oscillations as long as the coherence of the wavefunctions is preserved. The time period of a Bloch oscillation is given by $\tau_{\mathrm{B}}=(e F d / h)^{-1}, d$ being the spatial period of the potential. The amplitude of the real-space oscillations is determined by the localization length $L=\Delta / e F$, with $\Delta$ denoting the zero-field width of the energy band (Bastard et al. 1989).

This semiclassical model neglects the dependence of the band structure and the wavefunctions on the electric field itself. Surprisingly, it turned out to be difficult to establish a firm quantum-mechanical basis for Bloch's and Zener's calculations. After decades of intense theoretical debates (Nenciu 1991) it is now accepted that Bloch states which are the eigenstates of the periodic potential in the absence of an electric bias field evolve as Houston functions (Houston 1940) when the field is switched on. The Houston description is based on a single-band model neglecting Zener tunnelling. In the Houston picture, the wavepacket oscillations modify the dipole matrix elements of the optical interband transitions. The matrix elements contain time-dependent phase factors oscillating with the Bloch period $\tau_{\mathrm{B}}$. The Fourier transforms of the matrix elements correspondingly have sidebands with spacing $2 \pi / \tau_{\mathrm{B}}$. Hence, in the frequency regime, the optical interband absorption exhibits a series of evenly spaced resonances, the Wannier-Stark (WS) ladder, with an energy separation $\Delta E=e F d$ between adjacent resonances.

Since both the WS and the Houston wavefunctions form complete sets of functions, any charge carrier wavepacket can be described in either base. Whatever the choice, in the fully quantum-mechanical picture Bloch oscillations are quantum-interference phenomena involving WS states. A wavepacket undergoes oscillations in space and time with a frequency $\nu_{\mathrm{B}}=e F d / h$, the same dependence as predicted by BlochZener's model (Bastard 1989).

When an electric field is applied across the periodic potential, the continuous interband absorption spectra break up into discrete WS resonances at a bias field $F_{\mathrm{c}}$ where the energy separation $\Delta E$ between adjacent WS resonances becomes larger than their linewidth. Neglecting inhomogeneous broadening, the linewidth is given by $\Gamma=2 \hbar / T_{2}$, with $T_{2}$ being the dephasing time constant for interband coherence. The condition $\Delta E>\Gamma$ for observability of WS resonances in the frequency domain is identical to $\omega_{\mathrm{B}} \frac{1}{2} T_{2}>1$, the condition for observation of at least a single Bloch oscillation cycle in time-domain interband measurements.

Bloch oscillations up to now have never been observed in natural crystalline solids. A real breakthrough could only be reached after Esaki \& Tsu proposed in 1970 to search for Bloch oscillations in artificial semiconductor superlattice structures consisting of alternating layers of semiconductors with different bandgaps (Esaki $\&$ Tsu 1970). The larger period $d$ of such structures as compared to the lattice constant of bulk materials leads to $\mathrm{THz}$ oscillation frequencies already at modest fields of several $\mathrm{kV} \mathrm{cm}^{-1}$. The dephasing time constant, on the other hand, remains rather high (on the order of a few ps) up to fields of several tens of $\mathrm{kV} \mathrm{cm}^{-1}$, because excitonic effects are not quenched by the applied field as in bulk solids. After progress in molecular-beam epitaxy allowed the growth of high-quality samples, WS ladders could unambiguously be identified in 1988 in $\mathrm{cw}$ photocurrent and photoluminescence 


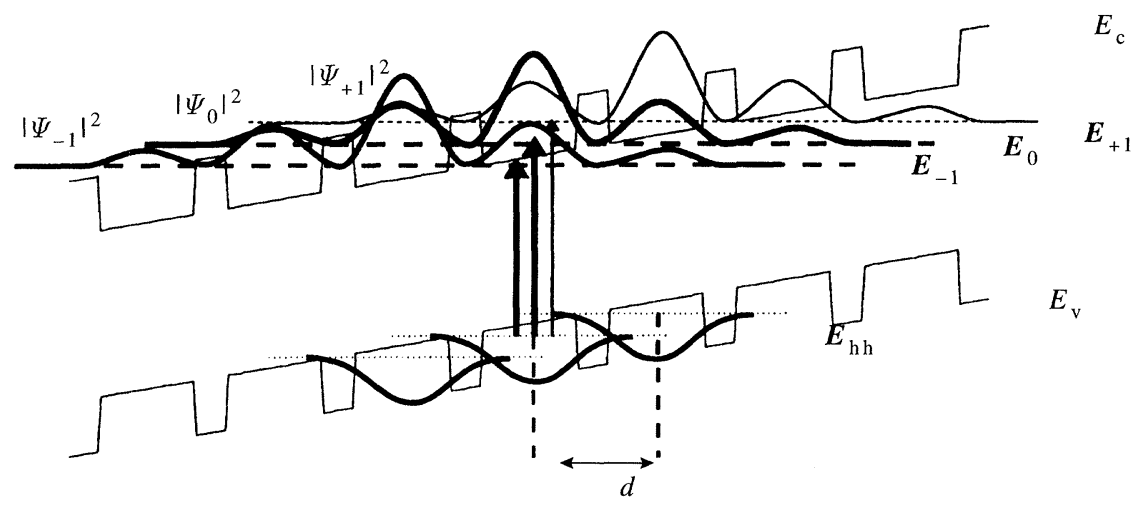

Figure 1. Scheme for optical excitation of Bloch oscillations in a superlattice biased into the WS regime. Only heavy-hole transitions are indicated.

spectra of GaAs/AlGaAs superlattice structures (Bleuse et al. 1988; Mendez et al. 1988). In 1990, the observation of negative differential velocity of electrons provided a first hint towards Bloch oscillations (Sibille et al. 1990). The first direct evidence was found in degenerate four-wave-mixing (FWM) experiments, where quantum beats by Bloch oscillations were observed in the nonlinear interband response (Feldmann et al. 1992; Leo et al. 1992).

\section{Optical excitation of Bloch oscillations}

Bloch oscillations are induced optically by simultaneous excitation of at least two WS ladder resonances with an ultrashort (broadband) light pulse, as illustrated in figure 1. The superlattice is biased into the WS regime for the first electron miniband. Under these bias conditions, the heavy-hole wavefunctions are fully localized in single wells whereas the electron and the light-hole wavefunctions, due to their lower effective mass, still extend over several wells. Upon excitation of heavy-hole exciton transitions, Bloch oscillations develop as quantum interference of the excited electron WS states.

Esaki \& Tsu (1970) proposed to utilize Bloch oscillations as a source for tunable ultrahigh-frequency electromagnetic signals. Bloch oscillations are associated with a quasi-static dipole moment coupling to electromagnetic waves (quasi-static used in the same sense as intraband: referring to transitions between WS states of the same miniband). The induced quasi-static macroscopic polarization density $P(t)$ is determined by the envelopes of the wavefunctions (Luo et al. 1994). In the singleparticle picture, i.e. neglecting excitonic effects, it can be calculated for a situation as that depicted in figure 1 (interband transitions from heavy-hole valence band states $|0\rangle$ into WS ladder states of the first electron miniband) by

$$
P(t)=\frac{e W}{V} \sum_{k}\left\{\sum_{i}\left[\rho_{i i}(t)\left(z_{00}-z_{i i}\right)\right]-2 \sum_{i>j>0}\left[z_{i j} \operatorname{Re}\left(\rho_{i j}(t)\right)\right]\right\} .
$$

$z$ is the spatial coordinate in the growth (and electric-bias-field) direction. $z_{i j}$ is related to the intraband dipole matrix element $\mu_{i j}$ according to $\mu_{i j}=-e z_{i j}=$ $-e\langle i|z| j\rangle$, with $i, j>0$ denoting WS ladder states. $\mu_{00}=-e z_{00}$ is the heavy-hole (hh) dipole moment. $\rho_{i j}(t)$ denotes the intraband density matrix elements, $\rho_{00}(t)$ is the heavy-hole population density. The summation is performed over the in-plane 
$\boldsymbol{k}$-vectors. $W$ denotes the number of periods of the superlattice. Assuming $\delta$-functionlike excitation of the levels, we can express the density-matrix elements up to the second order in the optical field $E_{0}$ by

$$
\begin{aligned}
& \rho_{i j}(t)=\frac{e^{2}\left\langle i\left|\boldsymbol{E}_{0} \boldsymbol{r}\right| 0\right\rangle\left\langle 0\left|\boldsymbol{E}_{0} \boldsymbol{r}\right| j\right\rangle}{\hbar^{2}} \mathrm{e}^{-\mathrm{i}\left(\omega_{i j}-\mathrm{i} \gamma_{i j}\right) t} \Theta(t), \quad i \neq j, \\
& \rho_{i i}(t)=\frac{e^{2}\left|\left\langle i\left|\boldsymbol{E}_{0} \boldsymbol{r}\right| 0\right\rangle\right|^{2}}{\hbar^{2}} \Theta(t) .
\end{aligned}
$$

Here, the longitudinal relaxation times are assumed to be infinitely long. $\gamma_{i j}$ is the dephasing rate between levels $i$ and $j, \Theta(t)$ is the Heaviside step function.

The polarization $P(t)$ induces a time-dependent local field change in the superlattice that can be probed electro-optically. Additionally, one expects emission of an electromagnetic transient with an electric field $E(t) \propto \partial^{2} P(t) / \partial t^{2}$. Both the local field change and the emitted radiation have two contributions of $P(t)$ with different temporal characteristics.

(1) Both terms in equation (3.1) contain expressions with a step-function-like time dependence. In the internal-field-probing measurements, these components produce an abrupt signal change, whereas they reveal themselves as a spike in the emitted electromagnetic signal lasting only during the optical excitation. This so-called instantaneous polarization signal is a consequence of the photocreation of electrons and holes with a field-induced spatial offset with respect to each other (Chuang et al. 1992).

(2) The second term in equation (3.1), an expression with oscillatory time dependence, describes the coherent charge oscillations. They are associated with an intraband polarization provided two conditions are fulfilled: (i) the interband transitions for the excitation of the upper levels must be allowed, i.e. $\left\langle i\left|\boldsymbol{E}_{0} \boldsymbol{r}\right| 0\right\rangle \neq 0$ for at least two values of $i$, in equations (3.2) and (3.3); (ii) the intraband dipole moments $\mu_{i j}=-e z_{i j}$ in equation (3.1) must have non-vanishing values. When both conditions are satisfied, local field oscillations and $\mathrm{THz}$ emission are expected with oscillation frequencies determined by the energy separations between the upper states, i.e. $\hbar \omega_{i j}=\Delta E_{i j}$. It is noteworthy that the effective masses of the charge carriers do not enter into the oscillation frequencies. One expects the same frequencies whether electrons, heavy holes or light holes are considered. The duration of the intraband charge oscillation is given by the dephasing time constant $\gamma_{12}^{-1}$.

Measurements of the internal field dynamics are performed by transmittive electrooptical sampling (TEOS) (Dekorsy et al. 1994a,b). Figure 2 displays the setup for this pump-probe measurement technique. Bloch oscillations are excited by linearly polarized pump pulses. Changes of the internal electric field along the growth direction of the superlattice are detected with circularly polarized probe pulses by comparing the intensities of the two perpendicularly polarized parts of the beam. Simultaneously to the TEOS signal, the nonlinear FWM signal diffracted into the $2 \boldsymbol{k}_{2}-\boldsymbol{k}_{1}$-direction can be recorded (Leisching et al. 1994, 1995).

Figure 3 displays the experimental setup for the detection of coherent electromagnetic radiation emitted from Bloch oscillations (Waschke et al. 1993; Roskos 1994). The radiation field is detected in the reflection direction with an optically gated photoconductive dipole antenna.

Both spectroscopic techniques utilize a self-modelocked Ti : sapphire laser system as source of tunable light pulses with a duration of 40-150 fs. The high pulse repetition rate of $76 \mathrm{MHz}$ together with the fact that both field amplitude and polarization 


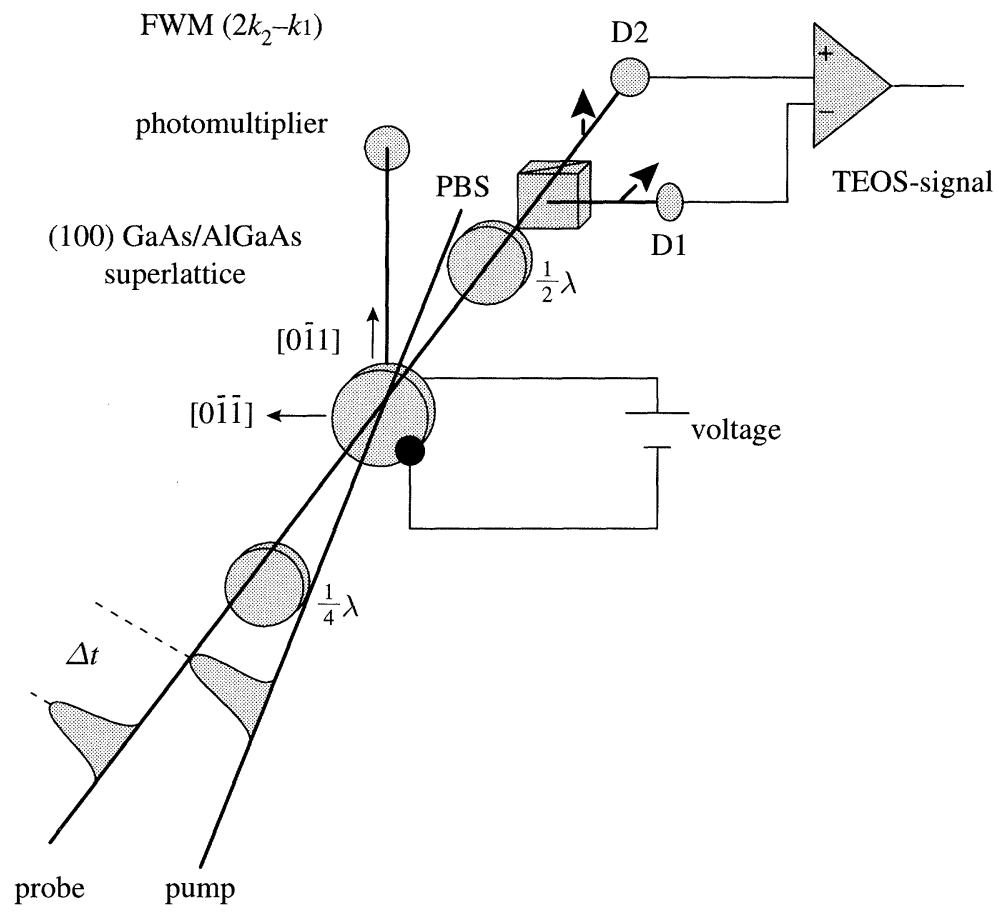

Figure 2. Experimental TEOS setup for the time-resolved detection of internal field changes.

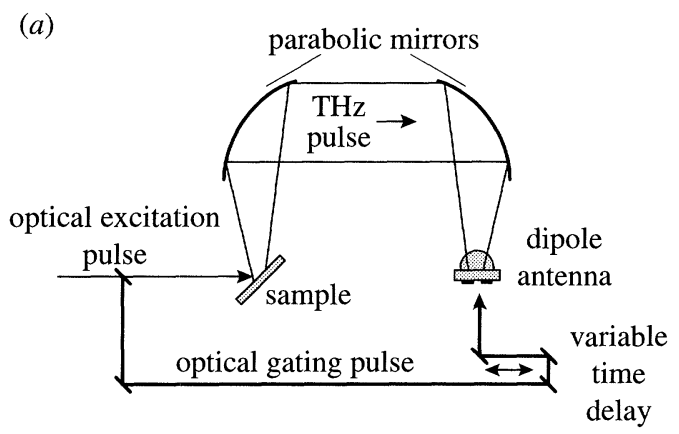

(b)

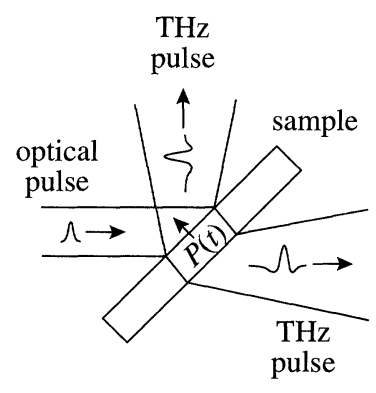

Figure 3. (a) Experimental setup for THz-emission spectroscopy. (b) Emission geometry indicating the orientation of the intraband polarization.

are measured, result in an exclusive sensitivity for coherent signals; all incoherent contributions average out effectively. The upper boundary for the frequency detectable with TEOS exceeds $10 \mathrm{THz}$ and is only limited by the duration of the optical pulses. The frequency limit of $\mathrm{THz}$-emission spectroscopy is approximately $4 \mathrm{THz}$, determined by the temporal response of the antenna.

In the following, data are presented for three superlattice structures consisting of 35 periods of $17 \AA$ wide $\mathrm{Al}_{0.3} \mathrm{Ga}_{0.7} \mathrm{As}$ barriers and GaAs wells with a width of 67, 97 and $111 \AA$, respectively (Leisching et al. 1994). The structures will be denoted in the following by the energy width $\Delta$ of the first electron miniband estimated by Kronig-Penney calculations to be 13,18 and $36 \mathrm{meV}$ for decreasing well width. The superlattice structures are grown by molecular-beam epitaxy on $\mathrm{n}^{+}$doped GaAs substrates. The superlattices are sandwiched between a $2500 \AA$ thick 


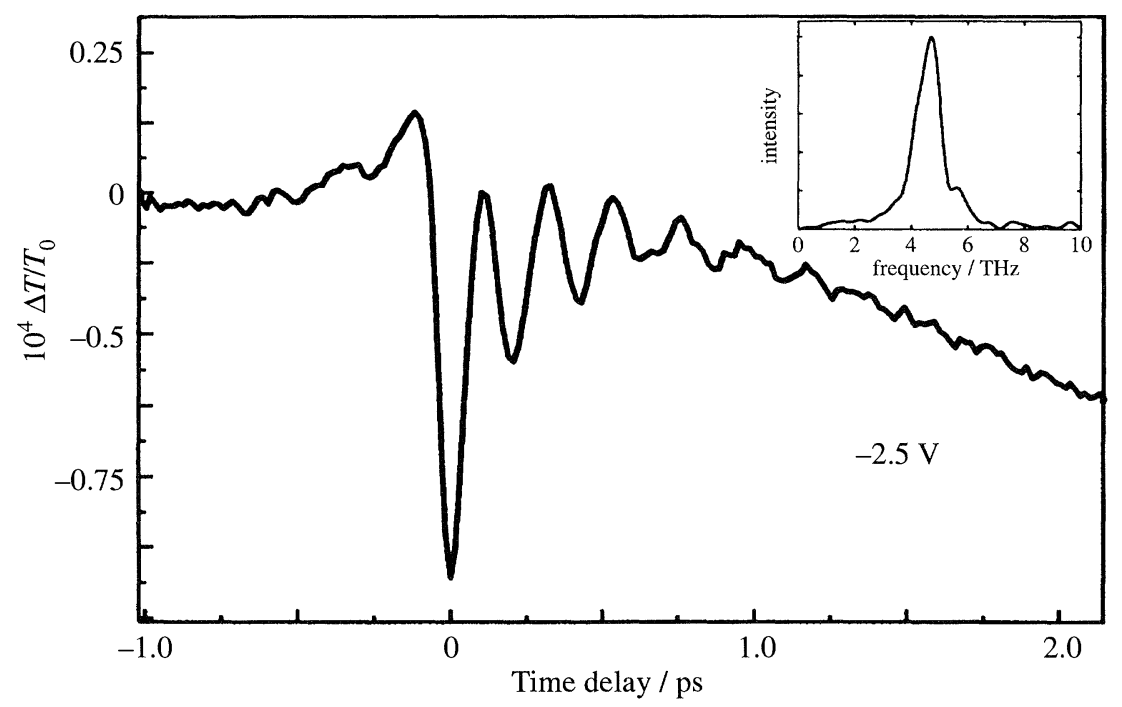

Figure 4. TeOs data recorded at $10 \mathrm{~K}$ at a superlattice with $36 \mathrm{meV}$ first electronic miniband width. The inset depicts the Fourier transform of the extracted oscillations only.

undoped $\mathrm{Al}_{0.3} \mathrm{Ga}_{0.7}$ As buffer on the substrate and another $3500 \AA$ thick buffer on top. A reverse-bias field can be applied between the substrate and a semitransparent $\mathrm{Cr}$ Schottky contact on top of the sample. For the internal-field-probing measurements, the substrate is removed by selective etching to allow transmission measurements. The THz-emission experiments are performed in reflection geometry under $45^{\circ}$ illumination without substrate removal.

\section{Experimental results}

(a) Internal field dynamics in a wide-bandgap superlattice at $10 \mathrm{~K}$

Figure 4 displays a TEOs transient for a superlattice with a width $\Delta$ of the lowest electronic miniband of $36 \mathrm{meV}$ for an applied reverse bias voltage of $-2.5 \mathrm{~V}$. The sample temperature is $10 \mathrm{~K}$. The pump pulse has $40 \mathrm{fs}$ duration corresponding to a spectral width of $40 \mathrm{meV}$. The sheet density of the photoexcited charge carriers is kept at $2 \times 10^{9} \mathrm{~cm}^{-2}$ per well, low enough that carrier-carrier scattering is insignificant on the time scale of interest of several picoseconds. The time-domain data reveal an initial step-like feature at the moment of the optical excitation. The step-like structure is explained as the contribution of the instantaneous polarization associated with the photogeneration of polarized electron-hole pairs. For longer time delays, the signal is strongly modulated by periodic oscillations, which can be traced for about $1.5 \mathrm{ps}$. On a picosecond time scale, the signal reveals a linear increase, which is attributed to incoherent carrier currents screening the internal field. The inset of figure 4 depicts the numerical Fourier transform of the oscillations extracted from the time-domain data by subtracting the background contribution due to the incoherent current. The Fourier spectrum peaks at $4.65 \mathrm{THz}$.

The dependence of the oscillations on the applied voltage is depicted in figure 5 . The tuning range is from $800 \mathrm{GHz}$ to $8 \mathrm{THz}$. The upper limit is close to the miniband width $(8.8 \mathrm{THz})$ and the optical phonon resonance of GaAs $\left(\nu_{\mathrm{LO}}=8.8 \mathrm{THz}\right)$. The lower frequency limit is basically given by the homogeneous broadening of the WS 


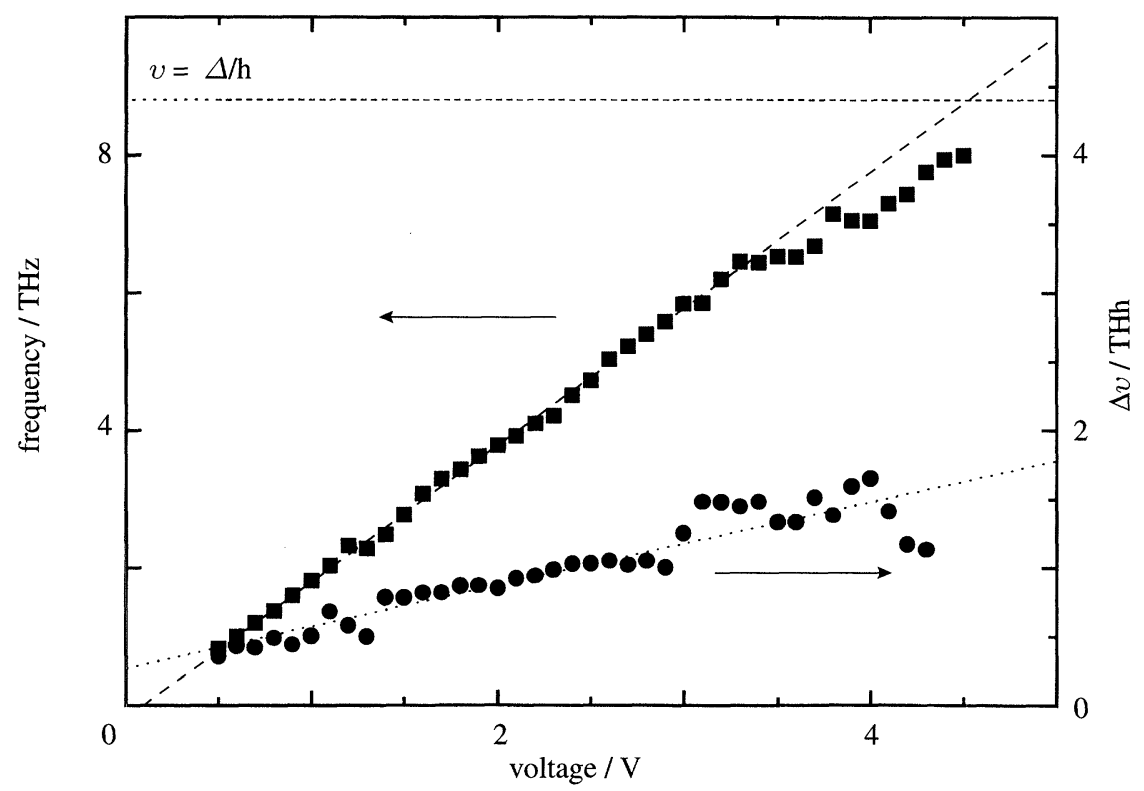

Figure 5. Frequency and linewidth of the Bloch oscillations plotted against the reverse bias voltage. The frequency is obtained from the Fourier transform of time-domain data and the linewidth is the FWHM from Gaussian fits to the spectra. Also shown is a linear fit to the frequency (dashed line) and the linewidth (dotted line). The horizontal dashed line corresponds to the first electronic miniband width of $36 \mathrm{meV}(8.8 \mathrm{THz})$.

transitions (see detailed discussion later). The oscillation frequencies observed rise linearly with the applied voltage, i.e. with the internal electric field, as expected from Bloch oscillation theory as outlined above. The linear fit to the data has a slope of $(1.99 \pm 0.02) \mathrm{THz} \mathrm{V}^{-1}$. This slope is in excellent agreement with the Bloch relation $\nu_{\mathrm{B}}=e d\left(U+U_{0}\right) / h l_{\text {intr }}$, with $d$ being the spatial period of the superlattice, $U$ the applied voltage and $l_{\text {intr }}$ the length of the intrinsic region in the device structure. $U_{0}$ is an offset voltage of $-0.2 \mathrm{~V}$ accounting for the built-in Schottky field and the static field screening by charge carriers accumulated at the interfaces between the superlattice and the $\mathrm{Al}_{0.3} \mathrm{Ga}_{0.7}$ As buffer layers (Leisching et al. 1994). The theoretical slope calculated from the nominal sample parameters $e d / h l_{\text {intr }}$ is $2.02 \mathrm{THz} \mathrm{V}^{-1}$ in good agreement with the experiment. This agreement is also a first hint of the observation of electronic coherence and not the dynamics of excitons, since for excitonic excitations a deviation from the simple $e F d$-dependence is expected due to the field dependence of the exciton binding energy (Leisching et al. 1995). The relevance of this point will be discussed later.

Figure 5 also displays the linewidth $\Delta \nu$ of the Fourier transforms (FwHM of Gaussian fits). At a frequency of $1 \mathrm{THz}$, the decay time constant of the coherent oscillations is approximately 2 ps. The decay time decreases linearly with increasing voltage and approaches a minimum of $650 \mathrm{fs}$ at $7 \mathrm{THz}$. Two major reasons are responsible for the observed dephasing times and their voltage dependence. (i) The dephasing is strongly influenced by the inhomogeneous broadening of the linewidth due to the sample growth and the internal electric field. These inhomogeneities lead to destructive interference of TEOS signals probed at different volume segments in the sample. Destructive interference affects high-frequency signals more strongly than low-frequency transients where the decay may even approach the homogeneous limit 
determined by interface roughness scattering (estimated decay time: 5 ps) and acoustic phonon scattering (estimated decay: 10 ps and above). (ii) A second reason for the decreasing dephasing time is the effect of alloy scattering of the wavepacket when it tunnels back and forth through the barriers. Given the assumption that each tunnelling process through the barriers leads to a certain destruction of the coherence, a shortening of the dephasing time is expected when more tunnelling events take place in the same time interval. This effect would give rise to a linear increase of the dephasing time. For frequencies above $7 \mathrm{THz}$, we observe that the dephasing time rises again and that the frequency starts to deviate from the $e F d$ relation. We attribute these effects to the direct coupling of Bloch oscillations to the LO phonon. Details will be published elsewhere (Dekorsy et al. 1996).

It is pointed out that the observed decay times are not yet limited by LO-phonon emission expected to become significant when the miniband width $\Delta$ significantly exceeds the LO-phonon energy of $36 \mathrm{meV}$ (von Plessen et al. 1994). The decay times observed here correspond to those of experiments on samples with a smaller value of $\Delta$, e.g. $T_{2}=2.8$ ps obtained from TEOS experiments in a superlattice with $18 \mathrm{meV}$ first electronic miniband width.

The linewidths of the Fourier spectra $\Delta \nu=1 / T_{2}$ at low frequencies are smaller than expected from the linewidth $\Gamma$ of the WS resonances as measured in cw photocurrent and transmission measurements. With the relation $\Gamma=2 \hbar / T_{2}$ for homogeneous lines, one estimates a value of $\Gamma$ of $0.66 \mathrm{meV}$ for a value of $T_{2}$ of 2 ps measured at the $1 \mathrm{THz}$ oscillation frequency. With an inhomogeneous contribution to $\Delta \nu$, the homogeneous width $\Gamma$ is even smaller than that value of $0.66 \mathrm{meV}$. In the cw measurements, one finds linewidths $\Gamma_{\text {inter }}$ of the transitions in the WS ladder of $1.5 \mathrm{meV}$ and above. In other words, predictions of the decay time constant of the coherent signal by the much-applied relation $\Gamma_{\text {inter }}=2 \hbar / T_{2}$ underestimate the measured decay time constant by at least a factor of two and by as much as a factor of four. In the next section we will show that this discrepancy arises from the different dynamics of the intraband and interband transitions observed in the different experiments.

The dashed horizontal line in figure 5 represents the cut-off frequency $\nu_{\mathrm{co}}=\Delta / h$. There the energy separation between adjacent WS states equals the miniband width $\Delta$. Above the cut-off frequency, the wavefunctions are strongly localized and no longer extend significantly into neighbouring wells. The overlap matrix element between adjacent WS states is so small that the wavepacket character of the excited WS states is practically lost. Our experiments show that the amplitude of the Bloch oscillations approaches zero at the cut-off frequency estimated by simple Bloch oscillation theory. The amplitude of the Bloch oscillations as a function of the electric field below the cut-off frequency $\nu_{\text {co }}$ is expected to decrease like $\Delta / e F$ due to the increased localization of the wavefunctions. Indeed, this behaviour is observed experimentally for higher fields (Dekorsy et al. 1994b). The decay of the maximum amplitude of the Bloch oscillations in our experiments is also influenced by the fact that the interband absorption strength of the WS states changes with increasing field. This leads to deviations from the expected amplitude-field relation, especially at low fields. However, the time-resolved experiments allow in principle the determination of the dynamic localization length of the wavepackets.

\section{(b) Interband versus intraband polarization}

In the preceding section, a closer analysis of the dephasing time constants suggests differences between the dephasing times of intraband and interband polarization 
quantities. In order to investigate these differences more systematically, TEOs measurements have been performed simultaneously with degenerate FWM measurements probing the nonlinear third-order interband polarization. Such measurements can be readily performed because it is possible to derive both the diffracted (FWM) signal and the TEOS from the same probe beam (see $\S 3$ ). Because also the same optical pump beam is utilized for excitation of the sample, identical excitation conditions and probing of the same sample volume ensure that the data obtained by the two techniques are fully comparable with each other.

The experiments are carried out on a sample with a $\Delta$ value of $18 \mathrm{meV}$ (Leisching et al. 1995). The spectrum of the $22 \mathrm{meV}$-wide pump pulses is centred on the spatially direct $h h$ exciton transition and covers several excitonic WS resonances as well as the onset of the respective exciton continua at the bias voltages relevant for the experiment. The density of excited electron-hole pairs is $2 \times 10^{9} \mathrm{~cm}^{-2}$.

At a voltage of $-0.5 \mathrm{~V}$ applied to the superlattice, the FWM data reveal an initial peak resulting from the interband polarization of the continuum states that is known to dephase on a $100 \mathrm{fs}$ time scale. The beating structure in the trailing part of the signal originates from the quantum interference of the excited excitonic transitions. From the signal decay, a dephasing time constant $T_{2 \text {,inter }}$ of 1 ps is deduced under the assumption of homogeneous broadening. The oscillation frequency observed is $2.2 \mathrm{THz}$. This result is in striking contrast to the respective values of the TEOS measurements performed under exactly the same conditions: an oscillation with a frequency of $2.6 \mathrm{THz}$ with a dephasing time of $2.4 \mathrm{ps}$ is observed under exactly the same experimental conditions. As a function of the applied voltage, we observe an increase of the frequency differences observed in both measurements, while the dephasing times stay approximately constant.

We attribute the differences in the frequencies to the differences of the excitonic interband transitions observed in FWM as compared to the energy splitting of electrons in continuum states observed in TEOS (Leisching et al. 1995). Under excitation of the $h h_{-1}$ and the $h h_{0}$ WS transition, the energy splitting of excitonic levels is smaller than $e F d$ due to the fact that the $h h_{-1}$ transition loses exciton binding energy with increasing localization, while the binding energy of the $h h_{0}$ transition is increased. The deviation from the $e F d$ value for a given field is in accordance with numerical calculations of the exciton binding energies (Leisching et al. 1995). In TEOs, the observed oscillations are dominantly performed by electrons in continuum states, which are unaffected by the electron-hole Coulomb interaction, thus the frequency of the oscillations obeys the $e F d$ relation, as also observed for the $36 \mathrm{meV}$ sample in figure 5. When we discuss the dephasing times, we come to the surprising result that the dephasing time of the electronic continuum states is more than a factor of two larger than the dephasing of the excitonic interband coherence and an order of magnitude larger than the dephasing of the interband coherence of continuum states. A reason for the difference between the excitonic interband coherence and the intraband coherence of the continuum states is attributed to the different coupling to the surrounding bath. For example it is feasible, that scattering events within the valence band destroy the interband coherence, while the coherence of the wavepacket in the conduction band is preserved. The difference of the interband dephasing of the continuum states compared to the electronic intraband coherence in the conduction band is even stronger. Again we can explain this difference by the same argument as above applied for the difference in the excitonic interband coherence and the intraband coherence. But this should only lead to a comparable difference in the de- 
phasing times. We conclude that scattering events that are phase destroying for the interband coherence of the continuum states do partially maintain the coherence of the electronic oscillations performed in the growth direction of the superlattice. One possible explanation is that scattering events involving momentum exchange within the $x-y$ plane do maintain the phase of the final state of the scattering event with respect to the motion along the growth direction. A similar scenario is feasible for carrier-carrier scattering with small momentum exchange in the $z$ direction, which destroys the interband coherence but partially maintains the intraband coherence.

\section{Bloch oscillations in a dissipative environment}

In this section, the influence of carrier-phonon scattering on the coherence is investigated. Experiments are discussed that allow to analyse selectively situations where emission, respectively absorption of LO phonons dominate carrier relaxation. The first case is realized by carrying out experiments at elevated sample temperature providing a background of incoherent LO phonons with a density determined by Bose-Einstein law. The second case, dominance of phonon emission, is realized by excitation of the charge carriers with enough excess energy that they can emit one or several optical phonons (hot excitation). As the latter experiments are carried out at low sample temperature, LO phonon absorption is insignificant compared to LO phonon emission immediately after optical excitation of the charge carriers. The main result of these experiments is that the intraband coherence of the wavepackets in the investigated superlattices can well survive one or several phonon-emission processes, but that the coherence is lost rapidly upon absorption of optical phonons.

\section{(a) Bloch oscillations at room temperature}

Bloch oscillations have been studied at elevated temperatures by FWM (Leisching et al. 1994) and THz-emission spectroscopy (Waschke et al. 1994). Here we demonstrate the observation of Bloch oscillations at room temperature by applying the TEOS technique (Dekorsy et al. 1995). In THz emission and FWM experiments the observation of Bloch oscillations at room temperature is aggravated by several reasons. The expected faster dephasing leads to a minimum in the minimum observable Bloch oscillation frequency of above $4 \mathrm{THz}$, which is above the present limit of conventional $\mathrm{THz}$ dipole antennas. In $\mathrm{FWM}$, the excitonic interband polarization will be rapidly destroyed due to the fact that the excitonic binding energy of approximately $4 \mathrm{meV}$ is well below the thermal energy $k T$ of $25 \mathrm{meV}$. Therefore, the absorption of LO phonons in addition to increased excitons and free carriers will lead to strong dephasing of the nonlinear excitonic polarization. Furthermore, higher electric fields have to be applied to overcome the critical field $F_{c}$. The necessary field strength is in the range where $e F d$ is much larger than the excitonic binding energy, so that excitons will experience a fast field ionization process.

We perform the room temperature experiments in the superlattice with $36 \mathrm{meV}$ miniband width and by applying laser pulses of $40 \mathrm{fs}$ pulse duration. Figure 6 depicts the oscillatory parts of TEOS signals recorded at room temperature. The increase of the Bloch oscillation frequency with rising reverse bias is clearly visible and the Fourier spectra reveal a linear field-voltage dependence expected for Bloch oscillations (Dekorsy et al. 1995). The amplitude decreases with increasing reverse bias due to the increase of localization. The dephasing times determined from fits to the time-domain data are in the range 130-200 fs. These fits show that the decay of the 


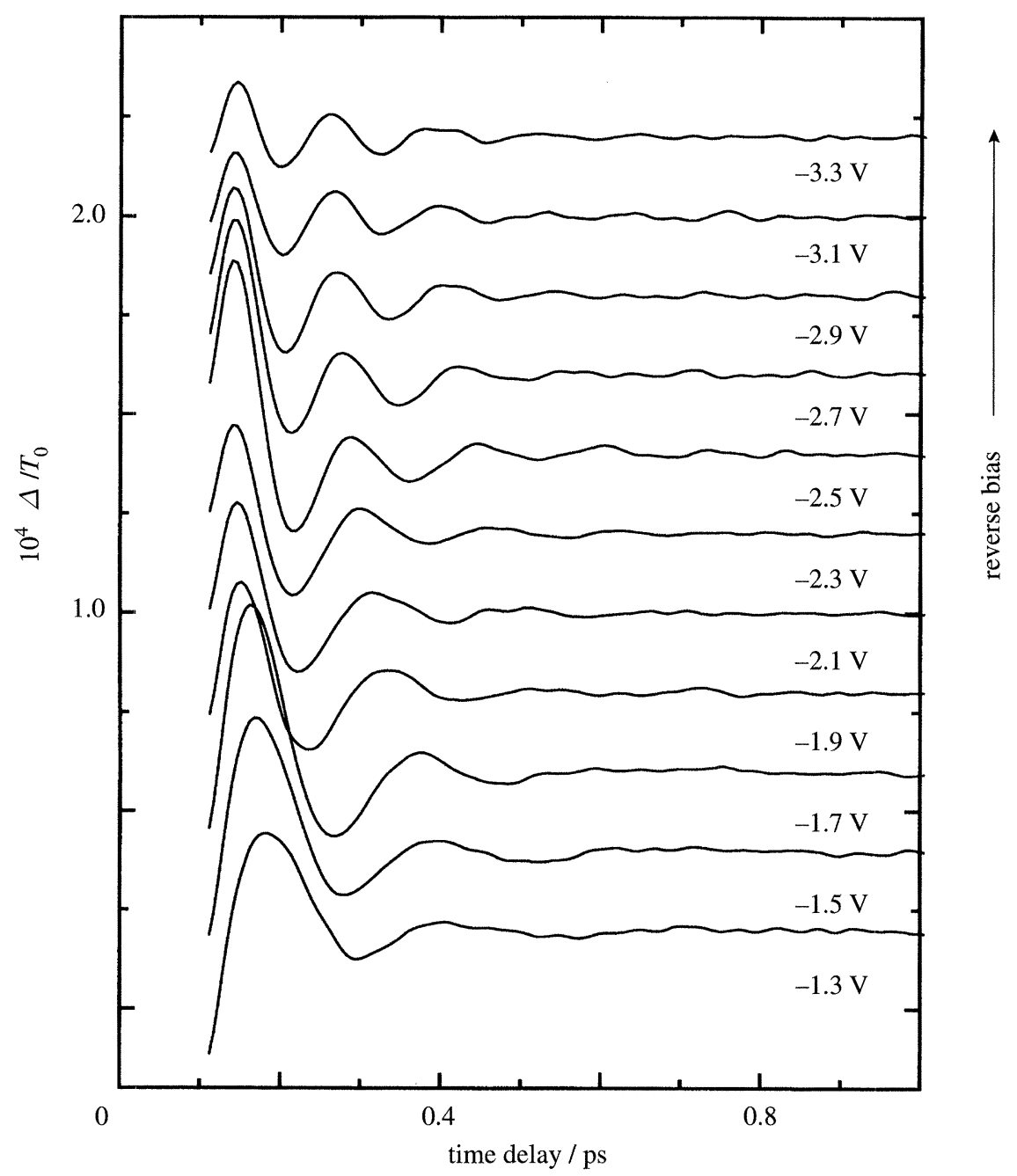

Figure 6. Extracted oscillatory contributions to TEOS recorded at room temperature in a superlattice with $36 \mathrm{meV}$ first electronic miniband width at different reverse bias voltages as indicated in the figure. The pulse duration is $40 \mathrm{fs}$ and the centre wavelength $845 \mathrm{~nm}$.

amplitude is not monoexponential and that the frequencies slightly shift with the time delay. The Fourier spectra (not shown) reveal a tunability from $4.5 \mathrm{THz}$ up to $8 \mathrm{THz}$ and show a strong asymmetry due to the non-exponential dephasing. The upper frequency is close to the limit given by the miniband width and the optical phonon resonance of GaAs. The lower limit is given by the requirement that the dephasing time at the same frequecy has to be larger than half an oscillation period.

In the sample under investigation we cannot resolve the WS ladder at room temperature due to the inhomogeneous broadening of the excitonic transitions. In addition, simultaneously performed FWM experiments reveal only an autocorrelation peak, i.e. the nonlinear interband polarization is destroyed within the pulse width. The comparison of the room temperature data to TEOS experiments performed at the same sample at $10 \mathrm{~K}$, as shown in figure 5 , gives an increase of the dephasing time by only a factor of three (Dekorsy et al. 1995). A stronger temperature dependence of the dephasing is expected for excitonic states (Kim et al. 1992), confirming 
our interpretation that the Bloch oscillations observed are performed by electrons in continuum sates. The observed dephasing time is attributed to the ultrafast thermalization of the excited distribution due to the absorption of LO phonons. Theoretical calculations for electron thermalization times due to LO phonon absorption are in the range of $600 \mathrm{fs}$ (Lam \& Singh 1995) and $200 \mathrm{fs}$ are determined experimentally for heavy holes (Kim et al. 1996). These electronic thermalization times are close to the observed value. In addition, simulations on the incoherent carrier transport dynamics within the superlattice show that the applied fields can be rapidly screened within several picoseconds at the carrier densities in our experiments (Dekorsy et al. 1996). This effect leads to transient and inhomogeneous fields and will manifest itself in a chirp (transient frequency) and an increased dephasing.

In cw photocurrent spectra of GaAs/AlAs and $\mathrm{GaAs} / \mathrm{Al}_{x} \mathrm{Ga}_{1-x} \mathrm{As}$ superlattices several groups have observed a WS ladder at room temperature in samples with large minibandwidth $(\Delta \sim 100 \mathrm{meV})$ with a minimum energy splitting of about $30 \mathrm{meV}$ (Fujiwara 1989; Mendez et al. 1990). By comparison with the spectra reported in the literature, we infer that the observed dephasing times are not the ultimate limit and that they can be extended by choosing different superlattice compositions. However, the minimum energy splitting of $18 \mathrm{meV}$ observed in our experiments are already well below $k T$ of $25 \mathrm{meV}$, so that we are close to the theoretical limit for the lower frequency limit given by LO phonon absorption.

\section{(b) Hot-carrier excitation}

In contrast to all experiments discussed previously, we now address measurements where the photogenerated charge carriers either have enough excess energy, or acquire it during Bloch oscillations, that they can relax by emission of optical phonons.

Figure 7 presents results of $\mathrm{THz}$-emission studies performed with a $13 \mathrm{meV}$ sample held at a temperature of $10 \mathrm{~K}$ and biased into the WS regime by an applied voltage of $-1.5 \mathrm{~V}$ (Roskos et al. 1994b). The density of the excited electron-hole pairs is $1 \times 10^{9} \mathrm{~cm}^{-2}$ for bandedge excitation but increases at higher photon energies because the energy of the optical excitation pulses is held constant. Oscillatory transients are observed not only for excitation of charge carriers at the excitonic bandedge resonances $(1.54 \mathrm{eV})$, but also for excitation into the band continua with photon energies as high as $1.642 \mathrm{eV}$, more than $80 \mathrm{meV}$ above the bandgap energy. At even higher energies, only single-cycle emission is observed. The decrease of the oscillation frequency with rising photon energy results from stronger quasi-static field screening for higher carrier density. Measurements of the bias-voltage dependence of the oscillation frequency (not shown) lead to the conclusion that the oscillatory signal results indeed from Bloch oscillations.

We now limit the discussion to a specific photon energy range where electrons are only excited into WS states of the first electron miniband (bandedge of the second miniband: at $1.624 \mathrm{eV}$, exciton transitions somewhat lower) but have enough excess energy that they can emit one or two LO phonons. In this case, it is known that the excited heavy holes (being in the first heavy-hole miniband) are so strongly localized that they do not contribute to a quantum-interference signal. Light holes, on the other hand, may contribute to the signal, but neglecting the complications of the light-hole band structure, their effective mass is similar to that of electrons and one can assume that the coherent dynamics of light holes is not much different from that of electrons.

Single-exponential fits of the time-domain data give decay time constants of the 


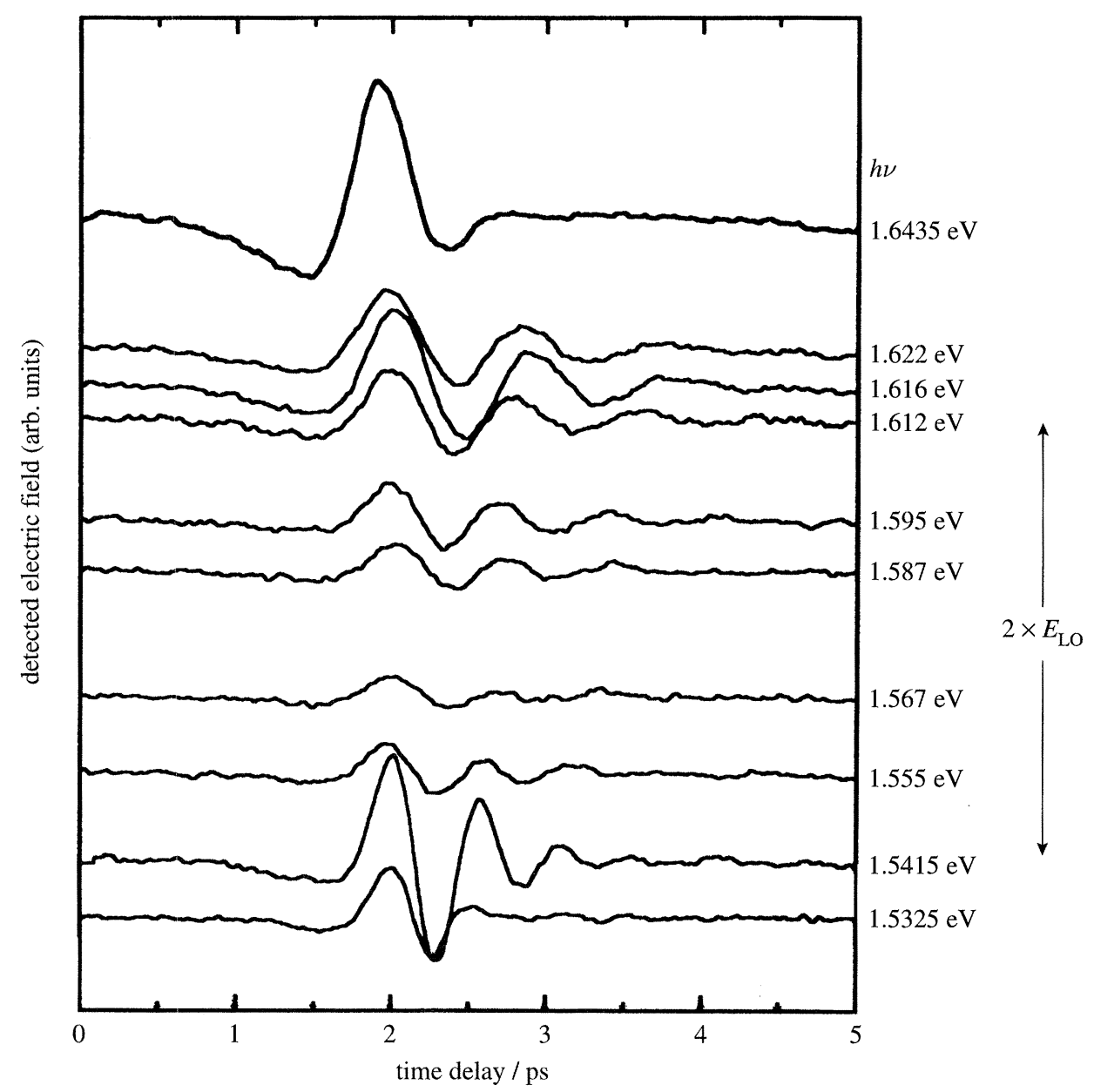

Figure 7. THz transients detected after optical excitation at different photon energies of the excitation pulses. The respective photon energies are given on the right-hand side of the panel.

Bloch oscillations of $0.75-1.25$ ps for both bandedge and hot excitation. In order to verify that the intraband phase coherence is preserved upon LO-phonon emission, white-light transmission measurements of the incoherent population lifetime of excited states, at least one LO-phonon energy above the bandedge, are performed (Wolter et al. 1996). In these experiments, the time evolution of the population at the edge of the conduction band is probed upon excitation of higher-lying conductionband states. The excess energy of the excited states is varied from below one LOphonon energy to above. The main result of the dual-wavelength pump-probe measurements is that the rise of the bandedge population becomes much faster when the excess energy of the electrons reaches the threshold of $36 \mathrm{meV}$ for LO-phonon emission (data not shown). Above threshold, the population rise time falls to 150$200 \mathrm{fs}$. This time constant is consistent with the well-established LO-phonon emission time in bulk GaAs that is expected to be similar to that of quasi-three-dimensional superlattices at low and medium bias.

The significant difference of the decay time of the coherent THz-radiation signal $(0.75-1.25 \mathrm{ps})$ and of the incoherent population lifetime of the excited states is clear 
evidence that the intraband coherence must be preserved at least partially during LO-phonon relaxation of the electrons. This is a surprising result because one expects loss and even destruction of coherence upon LO-phonon emission because the emission is accompanied with a momentum transfer that should equilibrate the $\boldsymbol{k}$-space distribution of the electrons and thus reduce or destroy the intraband polarization.

The reasons for phase conservation are elucidated by recent Monte Carlo simulations addressing the role of LO-phonon emission on the phase of the intraband polarization in our superlattice structures (Meier et al. 1995). The simulations reproduce the measured dependence of the $\mathrm{THz}$ waveform on the excess energy of the excited electrons quite well. The strongest $\mathrm{THz}$ signal is expected for bandedge excitation. The calculations predict, however, a Bloch-oscillation signal for all excess energies of the electrons although the carriers, if they have enough excess energy, undergo LO-phonon relaxation with a time constant of about 150-200 fs. The simulations show that the survival of phase coherence has several reasons. One is the polar character of LO-phonon emission that favours the transfer of small momenta and limits $\boldsymbol{k}$-space randomization of the electron distribution. Another reason for phase preservation is energy conservation that results in phase-space constraints by limiting the available final states in the scattering process.

\section{Outlook for technical implementations}

The results discussed above clearly suggest applications of Bloch oscillations in superlattice structures for practical voltage-tunable $\mathrm{THz}$-frequency emitters. The concept closest to our experimental situation is that of a coherent emitter driven by ultrashort optical pulses, as in the experiments. For technical purposes, the light source should be compact, cheap and reliable. Such sources will be available in the near future, most likely in the form of solid-state lasers, such as self-modelocked Ti:sapphire lasers pumped by diode lasers. To be of practical interest, the $\mathrm{THz}$ emitters must become more efficient than they are now. One estimates that the time-averaged power of the $\mathrm{THz}$ radiation (consisting of a 2 ps pulse every $13 \mathrm{~ns}$ ) is typically $0.1 \mathrm{nW}$, corresponding to a cw power of $1 \mu \mathrm{W}$ if the emission were continuous. Although this power level may be of interest for some purposes (especially if one considers the advantages of the full coherence of the radiation), higher intensities are highly desirable.

In all basic investigations up to now, the excitation density has been kept low to avoid complications by carrier-carrier scattering in the analysis of the underlying physics. For higher excitation densities, a redesign of the superlattice samples is required to avoid problems of field screening by charge-carrier accumulation. Currently, measurements are carried out on pin structures without buffer layers providing clear evidence that the emission process is superradiant, i.e. that the coherent radiation results from collective phase-synchronized emission from an ensemble of dipoles (Dicke superradiance). Over a large range of excitation densities, the emitted power increases quadratically with the density of photogenerated charge carriers instead of linearly for incoherent radiation (Victor et al. 1994). At densities in the $10^{10} \mathrm{~cm}^{-2}$ regime, the emitted power saturates because of dynamical screening involving charge carriers that have lost coherence and drift to the electrodes. For a sheet density of $10^{10} \mathrm{~cm}^{-2}$ electron-hole pairs, an average separation of the electron and holes by $10 \mathrm{~nm}$ (equivalent to one period of the superlattice) results in a screening field on the order of $1 \mathrm{kV} \mathrm{cm}^{-1}$. With a bias field of $4 \mathrm{kV} \mathrm{cm}^{-1}$ for a Bloch oscillation fre- 
quency of $1 \mathrm{THz}$, a considerable part of the field is screened already during the dephasing time of the intraband polarization. As a consequence, dynamical field screening imposes a fundamental limit to the strength of superradiant emission.

Superradiant emission, however, allows amplification of radiation by phase-correct feedback of the wave into the superlattice pumped by a second optical pulse. Neglecting absorption and other losses, the radiative gain for an ensemble of $N$ coherent dipoles (without population inversion) is the same as that of a completely population-inverted incoherent ensemble of $N$ two-level quantum systems. If amplification is achieved with the help of an external cavity, a concept of a synchronously pumped coherent $\mathrm{THz}$ emitter, i.e. a solid-state maser, can be envisaged.

So far, we have only discussed approaches that are derived directly from our experimental setup. For a sucessful technical implementation, the optically controlled emission should be replaced by electrical wavepacket injection. It has to be pulsed in synchronization with the charge oscillations. One can envisage devices with an injector region and a separately biased superlattice oscillation region. The injector region generates repetitive bunches of electrons that after transfer into the oscillation region provide tunable Bloch radiation. To realize a practical device many obstacles remain to be overcome but considering that a few years ago it would have been unthinkable to implement optically driven 'Esaki-Tsu' $\mathrm{THz}$ oscillators, there is now hope to achieve that goal.

We acknowledge contributions of P. Leisching, C. Waschke, F. Wolter, P. Haring Bolivar, H. J. Bakker, R. Ott, A. M. T. Kim, G. C. Cho and K. Victor. We are grateful for the financial support by the DFG, the Volkswagen Foundation, the BMBF and the Alfried Krupp Foundation.

\section{References}

Bastard, G. \& Ferreira, R. 1989 Spectroscopy of semiconductor microstructures, NATO ASI Series, p. 333. New York: Plenum.

Bleuse, J., Bastard, G. \& Voisin, P. 1988 Electric-field-induced localization and oscillatory electro-optical properties of semiconductor superlattices Phys. Rev. Lett. 60, 220-223.

Bloch, F. 1928 Z. Phys. 52, 555.

Chuang, S. L., Schmitt-Rink, S., Greene, B. I., Saeta, P. N. \& Levi, A. F. J. 1992 Optical rectification at semiconductor surfaces. Phys. Rev. Lett. 68, 102-105.

Dekorsy, T., Leisching, P., Köhler, K. \& Kurz, H. 1994a Electro-optic detection of Bloch oscillations. Phys. Rev. B 50, 8106-8109.

Dekorsy, T., Leisching, P., Beck, W., Ott, R., Dhaibi, Y., Schwedler, R., Roskos, H.G., Kurz, H. \& Köhler, K. $1994 b$ Internal field dynamics of coherent Bloch oscillations in superlattices. Superl. Microstr. 15, 11-14.

Dekorsy, T., Ott, R., Kurz, H., \& Köhler, K. 1995 Bloch oscillations at room temperature Phys. Rev. B 51, 17 275-17278.

Dekorsy, T., Ott, R., Bakker, H. J., Kurz, H., Köhler, K. 1996 THz Boch oscillations in superlattices at room trmperature. Proceedings of Ultrafast Processes in Spectroscopy IX. New York: Plenum. (In the press.)

Dignam, M. M. \& Sipe, J. E. 1991 Exciton Stark ladder in semiconductor superlattices. Phys. Rev. B 43, 4097-4112.

Esaki, L. \& Tsu, R. 1970 Superlattice and negative differential conductivity in semiconductors. IBM J. Res. Develop. 14, 61-65.

Feldmann, J., Leo, K., Shah, J., Miller, D. A. B., Cunningham, J. E., Meier, T., von Plessen, G., Schulze, A., Thomas, P. \& Schmitt-Rink, S. 1992 Phys. Rev. B 46, 7252-7255.

Fujiwara, K. 1989 Room-temperature observation of Stark ladder formation in a short-period GaAs-AlAs superlattice under an electric field. Jap. J. Appl. Phys. 28, L1718-L1720. 
Houston, W. V. 1940 Acceleration of electrons in a crystal lattice. Phys. Rev. 57, 184-186.

Kim, A. M. T., Hunsche, S., Dekorsy, T., Kurz, H. \& Köhler, K. 1996 Time-resolved study of the intervalance-band thermaliztion in a GaAs quantum-well. Appl. Phys. Lett. 68, 2956-2958.

Kim, D.-S., Shah, J., Cunningham, J. E., Damen, T. C., Schäfer, W., Hartmann, M. \& SchmittRink, S. 1992 Giant excitonic resonance in time-resolved four-wave mixing in quantum wells. Phys. Rev. Lett. 68, 1006-1009.

Lam, Y. L. \& Singh, J. 1995 Carrier thermalization by phonon absorption in quantum-well modulators and detectors. IEEE J. Quantum Electron. 31, 923-926.

Leisching, P., Haring Bolivar, P., Beck, W., Dhaibi, Y., Bruggüggermann, F., Schwedler, R., Kurz, H., Leo, K. \& Köhler, K. 1994 Bloch oscillations of excitonic wave packets in semiconductor superlattices.Phys. Rev. B 50, 14 389-14 404.

Leisching, P., Dekorsy, T., Bakker, H. J., Kurz, H., \& Köhler, K. 1995 Exceptionally slow dephasing of electronic continuum states in a semiconductor. Phys. Rev. B 51, 18 015-18018.

Leo, K., Haring Bolivar, P., Brüggemann, F., Schwedler, R. \& Köhler, K. 1992 Observation of Bloch oscillations in a semiconductor superlattice. Solid State Communs 84, 943.

Luo, M. S. C., Chuang, S. L., Planken, P. C. M., Brener, I., Roskos, H. G. \& Nuss, M. C. 1994 Generation of terahertz electromagnetic pulses from quantum-well structures. IEEE J. Quantum Electron. JQE-30, 1478-1487.

Meier, T., Rossi, F., Thomas, P. \& Koch, S. W. 1995 Dynamic localization in anisotropic Coulomb systems: field induced crossover of the exciton dimension. Phys. Rev. Lett. 75, $2558-2561$.

Mendez, E. E., Agulló-Rueda, F. \& Hong, J. M. 1988 Stark localization in GaAs-GaAlAs superlattices under an electric field. Phys. Rev. Lett. 60, 2426-2429.

Mendez, E. E., Agulló-Rueda, F. \& Hong, J. M. 1990 Temperature dependence of the electronic coherence of GaAs-GaAlAs superlattices. Appl. Phys. Lett. 56, 2545-2547.

Nenciu, G. 1991 Dynamics of band electrons in electric and magnetic fields: rigorous justification of the effective Hamiltonians. Rev. Mod. Phys. 63, 91-127.

von Plessen, G., Meier, T., Feldmann, J., Thomas, P., Goosen, K. W., Kuo, J. M. \& Knopf, R. F. 1994 Influence of scattering on the formation of Wannier-Stark ladders and Bloch oscillations in a semiconductor superlattice. Phys. Rev. B 49, 14058-14061.

Roskos, H. G. 1994 Coherent emission of electromagnetic pulses from Bloch oscillations in semiconductor superlattices. In Festkörperprobleme, advances in solid state physics (ed. R. Helbig), vol. 34, pp. 297-315. Braunschweig: Vieweg.

Roskos, H. G., Waschke, C., Schwedler, R., Leisching, P. \& Köhler, K. 1994 Bloch oscillations in GaAs/AlGaAs superlattices after excitation well above the bandgap. Superlattices Microstruc. 15, 281-285.

Sibille, A., Palmier, J. F., Wang, H. \& Mollot, F. 1990 Observation of Esaki-Tsu negative differential velocity in GaAs-AlAs superlattices. Phys. Rev. Lett. 64, 52-55.

Victor, K., Roskos, H. G. \& Waschke, C. 1994 Efficiency of submillimeter-wave generation and amplification by coherent wave-packet oscillations in semiconductor structures. J. Opt. Soc. Am. B 11, 2470-2479.

Waschke, C., Roskos, H. G., Schwedler, R., Leo, K., Kurz, H. \& Köhler, K. 1993 Coherent submillimeter-wave emission from Bloch oscillations in a semiconductor superlattice. Phys. Rev. Lett. 70, 3319-3322.

Waschke, C., Leisching, P., Haring-Bolivar, P., Schwedler, R., Brüggemann F., Roskos, H. G., Leo, K., Kurz, H. \& Köhler, K. 1994 Detection of Bloch oscillations in a semiconductor superlattice by time-resolved terahertz spectroscopy and degenerate four-wave mixing. Solid State Electr. 37, 1321-1326.

Wolter, F., Roskos, H. G., Kurz, H. et al. 1996 (In the press.)

Zener, C. 1934 Proc. R. Soc. Lond. A 145, 523. 